\title{
Radiation effects on the spinal cord: is mitigation in the long run possible?
}

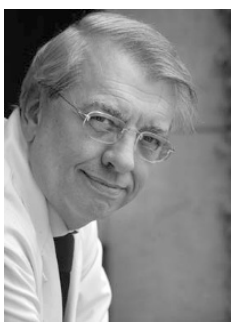

\author{
JJ Wyndaele \\ Antwerp, Belgium \\ E-mail: wyndaele@skynet.be
}

In this August issue of Spinal Cord you will find several interesting contributions.

Review: Wong et al. reminds us that the biologic response of the spinal cord after radiation is a continuously evolving process, as death of vascular endothelial cells and disruption of the blood-spinal cord barrier leads to a complex injury response resulting in demyelination and tissue necrosis. Cases of radiation myelopathy have reemerged with the increasing role of spine stereotactic body radiation therapy and re-irradiation. Experimental biologic strategies targeting the injury response pathways hold promise in mitigating the dreaded late effect of radiation treatment.

Animal research: Dong et al. demonstrated for the first time the expression of anaphylatoxins C5a, (which regulates the immune activation and takes part in other pathophysiological processes via binding with C5a receptor) after spinal cord ischemia reperfusion injury in rat. Hu et al. used a high-resolution synchrotron radiation-phase contrast tomography to better study the three-dimensional local blood angioarchitecture changes that occur after SCI supply to the spinal cord after an acute violent injury.

Diagnosis: Kim et al. showed that diffusion tensor imaging can be used to quantify the proximal and distal extents of spinal cord damage.

Pathophysiology: Invernizzi et al. found serum myostatin and serum sclerostin to be significantly higher in chronic SCI patients compared to healthy controls, making them potential biomarkers of muscle and bone modifications after SCI. Mohammadi et al. showed in a randomized, double-blind, placebo-controlled clinical trial that lipoic acid supplementation has no significant effect on measured inflammatory markers but it reduces fasting blood sugar, anthropometric parameters, food intakes, and blood pressure in men with chronic SCI.

Smoking: Saunders et al. found rates of smoking among persons with SCI to be well above national prevalence rates. They also found poorer access to care related to a greater likelihood of being a current smoker, no association with trying to quit smoking, but decreased likelihood of using cessation support among those who did attempt to quit.

Sleep disturbance: Spong et al. found people with chronic tetraplegia to experience more subjective sleep problems and worse quality of life than able-bodied counterparts. Treating the sleep disorders has the potential to improve their health and wellbeing.

Urology: Yildiz et al. demonstrated that repeated cystometric measurements in SCI patients with neurogenic detrusor overactivity lead to an increase in 1st unstable detrusor contraction and cystometric capacity. But such effect was seen when indwelling catheter and not when intermittent catheterization was used as bladder drainage.

Drugs: Krause et al. state that pain medication misuse must be of concern after SCI given its high prevalence among those with at least 1 painful condition and its relationship with pain indicators.

Reeducation: Rinkaewkanb and Kuptniratsaikul studied the effectiveness of inpatient rehabilitation in SCI patients in a centre in Thailand. The shorter duration between SCI and rehabilitation, a lower Barthel Index score on admission, the absence of co-morbidity and the ability to undertake an intensive rehabilitation program influenced positively the effectiveness of rehabilitation. Amatachaya et al. demonstrated prospectively that the ability on obstacle crossing in a closed/controlled environment is clearly correlated to intrinsic causes whereas a fall in an open environment may be associated with not only intrinsic but also extrinsic causes. This indicates the need to take care of both in order to manage risk of injury for the patients.

Education: Liu et al. investigated the difference in response to self-assessment questions in the original and an adjusted version of a sub-module of www.elearnSCI.org for student nurses.

Enjoy reading

Spinal Cord Series and Cases the best way to publish your case reports and small series/pilot studies. www.nature.com/scsandc.

Spinal Cord (2015) 53, 573; doi:10.1038/sc.2015.132 